\title{
Integrating 3D Surface Imaging with FIB/SEM Microscopy
}

\author{
B Volbert ${ }^{1}$, G Renka $^{1}, \mathrm{~K}$ Schock $^{1}$, A Lieb $^{2}$, M Dadras $^{3}$, S Kleindiek $^{1}$ \\ 1. Kleindiek Nanotechnik, Reutlingen, Germany \\ 2. Nanosurf AG, Liestal, Switzerland \\ 3. Centre Suisse d'Électronique et de Microtechnique, Neuchâtel, Switzerland
}

With the large amount of current research and development focused on nano wires, carbon nano tubes, and other nano scale materials, imaging these materials has become a large part of the challenges involved.

The two most prominent methods for imaging at the nano scale are Scanning Electron Microscopy (SEM) and Atomic Force Microscopy (AFM). These complimentary methods utilize fundamentally different principles for generating imagery - SEM exploits the interaction of electrons with matter, while AFM is based on physical interaction of a sharp tip with the sample surface.

Both approaches have strengths and weaknesses. The SEM's strength is to quickly generate images with a large range of magnifications, making it easy to locate the area of interest. However, it doesn't yield 3D information, e.g. "invisible" contamination layers. The AFM's main advantage is its ability to obtain 3D information, the downsides are that it is hard to find the target area and image generation is slow. Combining these two tools into one setup - putting an AFM inside an SEM - gives quick access to a more complete data set. Additionally, FIB-milled or FIB-deposited structures can be characterized using this combination of tools in a FIB/SEM system.

For the past twenty years, various groups have been working to integrate scanning probe microscopy methods into scanning electron microscopes, demonstrating both the need for - as well as the difficulty in - combining these methods [1-3].

In the past, the combination of these two imaging methods required cumbersome modifications to dedicated scanning electron microscopes. The novel approach described in this work yields a system that can be fitted to almost any commercially available SEM/FIB tool on the market. The utility of this combination of tools is demonstrated with several examples where locating the area of interest purely by AFM or light microscopy would have been highly impractical.

[1] DA Walters, Appl. Phys. Lett. 65 (1994) p. 787

[2] U Stahl, CW Yuan, AL de Lozanne, M Tortonese, Appl. Phys. Lett. 68 (1994) p. 2878

[3] K Fukushima, D Saya, H Kawakatsu, Jpn. J. Appl. Phys. 39 (2000) p. 3747

[4] A Altes, I Joachimsthaler,G Zimmermann, R Heiderhoff, Proceedings of the 9th International Symposium on the Physical and Failure Analysis of Integrated Circuits (IPFA 2002) p. 196. 


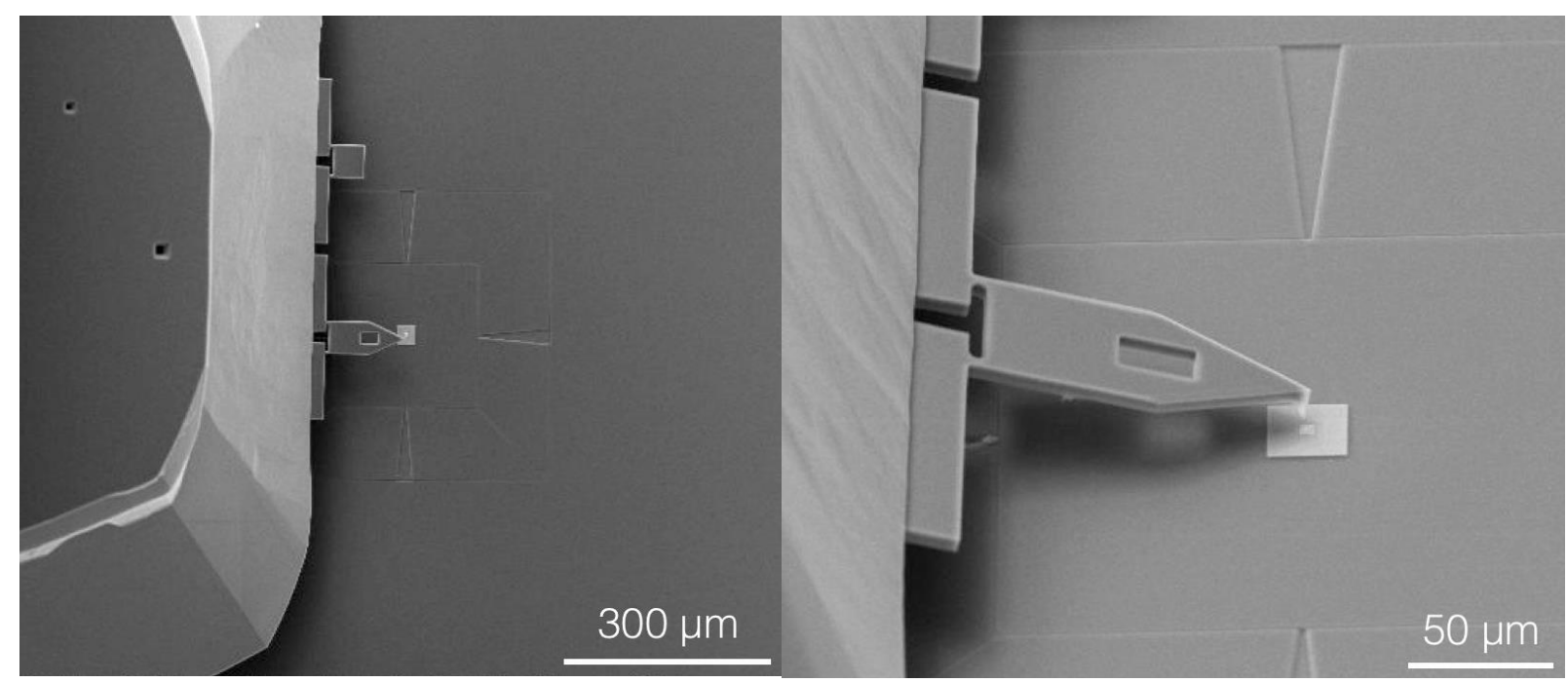

Figure 1. Addressing the target area for AFM inspection using the SEM.
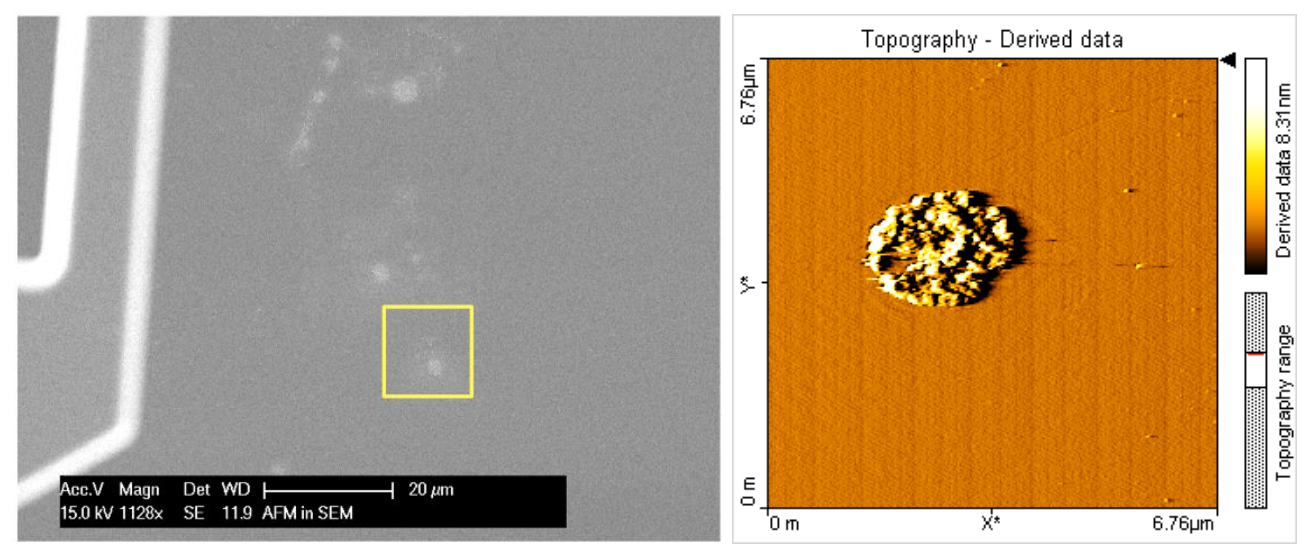

Figure 2. Si surface observation by SEM and AFM characterization of defects. The defects have a height of about $12 \mathrm{~nm}$. 